\title{
Glucagon-like peptide-1 modulates RAW264.7 macrophage polarization by interfering with the JNK/STAT3 signaling pathway
}

\author{
SHAN WAN and HUI SUN \\ Department of Endocrinology, Union Hospital, Tongji Medical College, \\ Huazhong University of Science and Technology, Wuhan, Hubei 430022, P.R. China
}

Received February 14, 2018; Accepted December 11, 2018

DOI: $10.3892 /$ etm.2019.7347

\begin{abstract}
Insulin resistance and metabolic disorders are closely associated with low-grade chronic inflammation. Aberrant macrophage activation to M1 or M2 is characterized by a deleterious state of chronic inflammation and loss of positive trophic signals. Glucagon-like peptide-1 (GLP-1) is used to treat diabetes due to its beneficial role against insulin resistance. The present study examined the effect of GLP-1 on macrophage activation, which contributed to M2 polarization and secretion of anti-inflammatory factors. In addition, the present study demonstrated that GLP-1 was able to reduce M1 polarization and inflammatory response by using the murine monocyte/macrophage cell line RAW264.7 and detecting M1/M2-specific genes. RAW264.7 cells were incubated with GLP-1 in the presence or absence of lipopolysaccharide or interleukin-4, the c-Jun N-terminal kinase (JNK) and signal transduction and transcriptional activation factor 3 (STAT3) activity was assessed by quantification of phosphorylation expression and macrophage polarization was determined by detecting M1/M2-specific genes expression. The results demonstrated that GLP-1/GLP-1 receptor attenuated the phosphorylation of JNK and its signal transduction through the cyclic adenosine monophosphate/protein kinase A signaling pathway, while the phosphorylation of STAT3 increased through following treatment with GLP-1. The present study observed that GLP-1 exerts its beneficial effects on macrophage polarization by modulating the JNK/STAT3 signaling pathway. The present results also suggested that the effects of GLP-1 on endocrine and metabolic diseases are possibly mediated by modulation of signaling pathways, and provide a basis for pharmacologic targeting of macrophage
\end{abstract}

Correspondence to: Dr Hui Sun, Department of Endocrinology, Union Hospital, Tongji Medical College, Huazhong University of Science and Technology, 1277 Jiefang Street, Wuhan, Hubei 430022, P.R. China

E-mail: 1114158713@qq.com

Key words: glucagon-like peptide-1, M1/M2 macrophage, inflammatory signaling, metabolism, signal transduction and transcriptional activation factor 3, c-Jun N-terminal kinase activation and an insight into the molecular mechanisms involved in the progression of metabolic diseases.

\section{Introduction}

Increasing evidence suggests that immune and inflammatory responses serve pivotal roles in obesity, diabetes and cardiovascular diseases $(1,2)$. Metabolic signals emerging from metabolic cells initiate inflammatory responses and damage metabolic homeostasis, thus leading to infiltration of macrophages into metabolic tissues, inflammation and insulin resistance (1). Macrophages are essential components of the innate immunity system, and serve a dynamic role in host defense, inflammation and tissue homeostasis (3). Monocyte/macrophages are the major source of inflammatory cytokines, which participate in the pathogenesis of diabetes, obesity and insulin resistance through paracrine and endocrine mechanisms (4-6).

The properties and activation state of macrophages change in different local environments, thus exhibiting an important heterogeneity (6). Under inflammatory and noninflammatory stimuli environments, macrophages display various status (3). M1 and M2 are two separate polarization activation states of macrophages, which are usually defined in vitro, while tissue macrophages may be activated at intermediate states between M1 and M2 (7-9). Proinflammatory mediators such as lipopolysaccharides (LPS) and interferon- $\gamma$ can induce M1 macrophages, which are also named 'classically activated' macrophages (7). M1 macrophages produce proinflammatory cytokines, including tumor necrosis factor- $\alpha$ (TNF- $\alpha$ ), interleukin (IL)-6 and IL-12, and generate reactive oxygen species such as nitric oxide (NO) via activation of inducible nitric oxide synthase (iNOS) (7). M2, or 'alternatively activated' macrophages, are generated in vitro by exposure to IL-4 and IL-13 (4). M2 macrophages have low proinflammatory cytokine expression and generate high levels of the anti-inflammatory cytokine IL-10 (4). Additionally, M2-polarized macrophages can enhance arginase production. This enzyme blocks iNOS activity by competing for the arginine substrate that is required for NO production (9). M2 macrophages are considered to block inflammatory responses and repair tissue during inflammatory responses as well as being involved in the promotion of tissue repair (7-9). Upon induction, macrophage state can switch from activated M1 state to M2 and vice versa (3,7-9). 
Glucagon-like peptide-1 (GLP-1) is secreted from intestinal L-cells and functions in nutrient ingestion. It is considered to have numerous glucose-lowering actions, including potentiating glucose-dependent insulin secretion, inhibiting glucagon secretion, enhancing $\beta$ cell growth, suppressing appetite and delaying gastric emptying (10-12). Additionally, GLP-1 appears to improve insulin sensitivity in patients with type 2 diabetes and animal models (13). Previous studies have demonstrated that GLP-1 reduces the accumulation of monocytes/macrophages and the expression of inflammatory mediators such as TNF- $\alpha$ and monocyte chemotactic protein in activated macrophages (14). A previous study demonstrated that GLP-1 reduces the numbers of M1 macrophages and the mRNA expression levels of M1 marker genes, and reduces the expression levels of inflammatory factors in adipose tissue and peritoneal macrophages (15). Considering that GLP-1 receptors are abundantly expressed in the surface of numerous cell types besides pancreatic islet cells, gastrointestinal cells, neural cells and mononuclear macrophages, GLP-1 may serve more important roles than expected. An in vitro study has elucidated that GLP-1 and GLP-1 agonists increase M2 macrophage-related markers and the secretion of the anti-inflammatory cytokine IL-10 when acting on human mononuclear macrophages, and has observed that GLP-1 induces macrophages into the M2 phenotype by signal transduction and transcriptional activation factor 3 (STAT3) activation (16). It is well known that STAT3 serves a key role in macrophage activation towards the M2 phenotype (17). As a repressor protein of the inflammatory response, STAT3 in resident macrophages acts as a transcription factor mediating the anti-inflammatory effects of IL-10 (18). STAT3 is the dominant mediator of the anti-inflammatory effects exhibited by IL-10, which acts to inhibit LPS-mediated TNF- $\alpha$ and IL-6 generation in macrophages (19). The effects of intracellular cAMP elevation on the production of inflammatory mediators in macrophages were originally reported to be mediated by protein kinase A (PKA) (20). Furthermore, cyclic adenosine monophosphate (cAMP) is a paramount factor for macrophage activation towards the M2 phenotype (20-22).

While the roles of STAT3 in macrophages are well supported, little is known about how GLP-1/GLP-1 receptor (GLP-1R) activates STAT3 signaling and the underlying mechanisms. With regard to macrophage polarization, the effects of GLP-1 on signal transduction have scarcely been documented to date, to the best of our knowledge. The present study elucidated that GLP-1R signaling contributes to the inhibition of JNK activation through the cAMP/PKA pathway, resulting in the activation of STAT3, which inhibits inflammation and M1 activation and promotes M2 activation. These findings suggest that modulations of signaling pathways are essential underlying mechanisms of GLP-1 on a broad spectrum of metabolic diseases.

\section{Materials and methods}

Reagents. Recombinant human GLP-1 (cat. no. 130-08) and murine IL-4 (cat. no. 214-14) were purchased from PeproTech EC Ltd. (London, UK). LPS was purchased from Sigma-Aldrich (Merck KGaA, Darmstadt, Germany). Enhanced BCA Protein Assay kit (cat. no. P0010S) was purchased from Beyotime Institute of Biotechnology (Haimen, China). Forskolin and $\mathrm{H} 89 / 2 \mathrm{HCl}$ were purchased from Selleck Chemicals (Houston, TX, USA). The anti-c-Jun N-terminal kinase (JNK; cat. no. 9252), anti-phosphorylated JNK (cat. no. 4668), anti-phosphorylated
Table I. Gene and primer sequences.

Gene $\quad$ Primer sequences

$\begin{array}{ll}\text { ARG-1 } & \text { Forward, 5'-CTCCAAGCCAAAGTCCTTAGAG-3' } \\ & \text { Reverse, 5'-AGGAGCTGTCATTAGGGACATC-3' }\end{array}$

MGL-1 Forward, 5'-TGAGAAAGGCTTTAAGAACT GGG-3' Reverse, 5'-GACCACCTGTAGTGATGTGGG-3'

MRC-1 Forward, 5'-TGGGCTACAGGAGAACCCAACTTT-3' Reverse, 5'-GCAGTGGCATTGATGCTGCTGTTA-3'

IL-10 Forward, 5'-GCTCTTACTGACTGGCATGAG-3' Reverse, 5'-CGCAGCTCTAGGAGCATGTG-3'

IL-6 Forward, 5'-ACAAAGCCAGAGTCCTTCAGAGAG-3' Reverse, 5'-TTGGATGGTCTTGGTCCTTAGCCA-3'

iNOS Forward, 5'-AATCTTGGAGCGAGTTGTGG-3' Reverse, 5'-CAGGAAGTAGGTGAGGGCTTG-3'

TNF- $\alpha$ Forward, 5'-TCTCAGCCTCTTCTCATTCCTGCT-3' Reverse, 5'-AGAACTGATGAGAGGGAGGCCATT-3'

GAPDH Forward, 5'-TGAAGCAGGCATCTGAGGG-3' Reverse, 5'-CGAAGGTGGAAGAGTGGGAG-3'

ARG, arginine-1; MGL-1, macrophage galectin-1; MRC-1, mannose receptor-1; IL, interleukin; iNOS, inducible nitric oxide synthase; TNF- $\alpha$, tumor necrosis factor- $\alpha$

STAT3 (cat. no. 9145), anti-STAT3 (cat. no. 4904) and anti-GAPDH antibodies (cat. no. 2118) were all obtained from Cell Signaling Technology, Inc. (Danvers, MA, USA). The Cyclic AMP EIA kit (cat. no. 581001) was purchased from Cayman Chemical Company (Ann Arbor, MI, USA).

Cells and cell culture conditions. RAW264.7 cells were provided by Professor Zheng (Wuhan Union Hospital, Wuhan, China), and were cultured in Dulbecco's modified Eagle's medium (DMEM)-high glucose (HG) medium (Hyclone; GE Healthcare Life Sciences, Logan, UT, USA) supplemented with $10 \%$ fetal bovine serum (Gibco; Thermo Fisher Scientific, Inc., Waltham, MA, USA) and antibiotics (100 U/ml penicillin and $100 \mathrm{U} / \mathrm{ml}$ streptomycin). Cells were maintained in an incubator at $37^{\circ} \mathrm{C}$ under a humidified atmosphere of $5 \% \mathrm{CO}_{2}$. RAW264.7 cells were seeded in 6-well plates at a density of $5 \times 10^{5}$ cells/well and the adherent cells were grown in serum-free DMEM-HG overnight for cell growth synchronization. Then, RAW264.7 cells were cultured with or without GLP-1 $(10 \mathrm{nM})$ for $24 \mathrm{~h}$ at $37^{\circ} \mathrm{C}$, followed by absence or addition of LPS $(100 \mathrm{ng} / \mathrm{ml})$ or IL-4 $(10 \mathrm{ng} / \mathrm{ml})$ for inducing polarization, and were preincubated at $37^{\circ} \mathrm{C}$ with the corresponding adenylyl cyclase activator Forskolin $(10 \mu \mathrm{M})$ or PKA inhibitor $\mathrm{H} 89 / 2 \mathrm{HCl}(10 \mu \mathrm{M})$ for 30 min prior to GLP-1 intervention.

Enzyme immunoassay (EIA) method to measure intracellular cAMP levels. RAW264.7 cells were treated at $37^{\circ} \mathrm{C}$ without or with GLP-1 $(0.5,1,5,10$ or $30 \mathrm{nM})$ for $8 \mathrm{~h}$, followed by incubation and solubilization with $0.1 \mathrm{M} \mathrm{HCl}$ at room temperature for $20 \mathrm{~min}$ to avoid degradation of cAMP at $37^{\circ} \mathrm{C}$. Upon centrifugation at $1,000 \mathrm{x} \mathrm{g}$ for $10 \mathrm{~min}$ at $4^{\circ} \mathrm{C}$, the cAMP content of the supernatant was quantified using the aforementioned Cyclic AMP EIA 

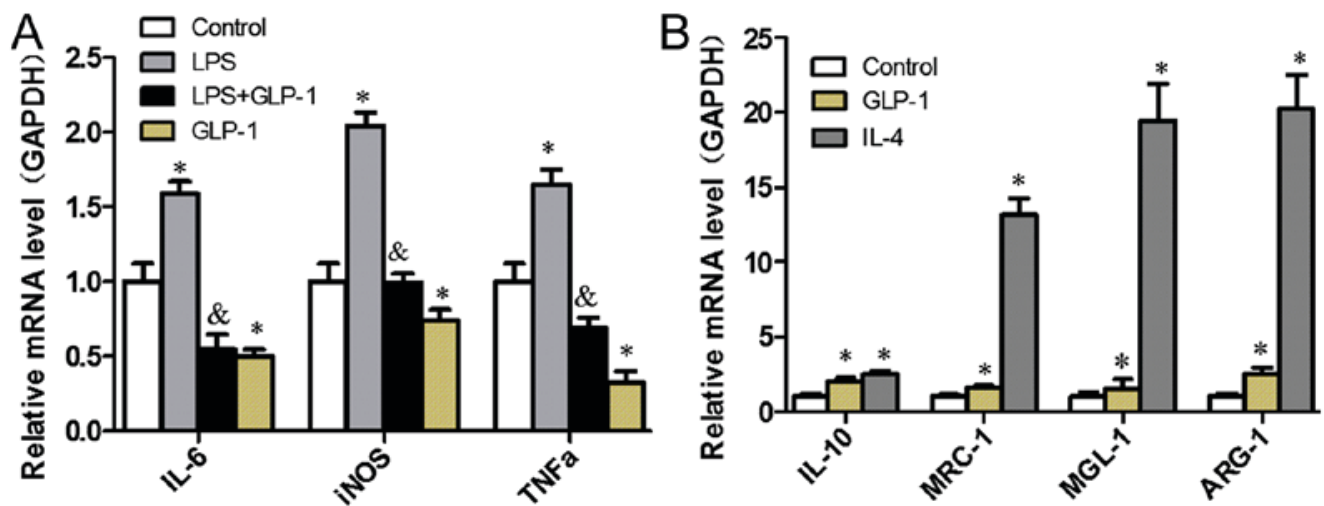

Figure 1. GLP-1 induces M2 polarization and inhibits M1 polarization in RAW264.7 cells. RAW264.7 cells were cultured with or without 10 nM GLP-1 for 24 h, followed by (A) LPS (100 ng/ml) stimulation or (B) IL-4 $(10 \mathrm{ng} / \mathrm{ml})$ incubation for $24 \mathrm{~h}$ to induce polarization. Then, the macrophages were used for isolation of total RNA. The mRNA expression levels of M1/M2-specific genes were determined by reverse transcription-quantitative polymerase chain reaction. The results are representative of three independent experiments. ${ }^{*} \mathrm{P}<0.05$ vs. control group; ${ }^{\circledR} \mathrm{P}<0.05$ vs. lipopolysaccharides group). GLP-1, glucagon-like peptide-1; LPS, lipopolysaccharide; IL, interleukin.

kit according to the manufacturer's instructions. Additionally, the Forskolin $(10 \mu \mathrm{M})$ group was used as a positive control. The optical density of the plate was read at a wavelength of $412 \mathrm{~nm}$ using a Spectrophotometer and an Absorbance Reader (each, Bio-Rad Laboratories, Inc.). cAMP concentration was calculated using a computer spreadsheet named EIADouble (2011) available for data analysis (Cayman Chemical Company).

Western blot analysis. Briefly, macrophages were solubilized and total protein was extracted with radioimmunoprecipitation assay lysis buffer including phosphorylated protease inhibitors $\mathrm{A}$ and $\mathrm{B}$ and phenylmethanesulfonyl fluoride (Beyotime Institute of Biotechnology). Upon centrifugation at $13,275 \mathrm{x}$ g at $4^{\circ} \mathrm{C}$ for $10 \mathrm{~min}$, the supernatant was collected and the protein content was quantified by employing a bicinchoninic acid protein assay kit. A total of $25 \mu \mathrm{g}$ protein per lane was separated by $8-10 \%$ SDS-PAGE and transferred onto a polyvinylidene difluoride transfer membrane (EMD Millipore, Billerica, MA, USA) by applying an electro-blotting apparatus. Then, the membranes were blocked with $5 \%(\mathrm{w} / \mathrm{v})$ skimmed milk in TBS with Tween-20 (TBST) for $1-2 \mathrm{~h}$ at $37^{\circ} \mathrm{C}$, followed by incubation overnight at $4^{\circ} \mathrm{C}$ with the following primary antibodies: Anti-STAT3 (1:2,000), anti-phosphorylated STAT3 (Tyr705; 1:2,000), anti-phosphorylated JNK (Thr183/Tyr185; 1:1,000) and anti-JNK (1:1,000). The membranes were also blotted with an anti-GAPDH antibody $(1: 1,000)$ serving as an internal calibration control. Subsequently, the membranes were washed with TBST and incubated with a horseradish peroxidase-labeled goat anti-rabbit immunoglobulin G (cat. no. GGHL-90P; 1:5,000; Immunology Consultants Laboratory, Inc., Portland, OR, USA) at room temperature for $1 \mathrm{~h}$. The protein bands were visualized by an enhanced chemiluminescence kit (Thermo Fisher Scientific, Inc.). The images were captured using the GelDoc XR System (Bio-Rad Laboratories, Inc.). Quantitative analysis of western blots was performed using ImageJ 1.4 software (National Institutes of Health, Bethesda, MD, USA).

Reverse transcription-quantitative polymerase chain reaction $(R T-q P C R)$. Total RNA was extracted from RAW264.7 cells with RNAiso Plus (Takara Bio, Inc., Otsu, Japan). RT was carried out using a PrimeScript RT Reagent kit (Perfect Real Time; Takara
Bio, Inc.) following the manufacturer's protocol. A total of $2 \mu \mathrm{g}$ total RNA was reverse-transcribed in a volume of $10 \mu 1$ for cDNA synthesis. The resulting cDNA was amplified using a SYBR ${ }^{\circledR}$ Premix Ex Taq (Tli RNaseH Plus) kit (Takara Bio, Inc.). qPCR was performed in the CFX96 ${ }^{\mathrm{TM}}$ Real-Time PCR system (Bio-Rad Laboratories, Inc.,). The PCR primers used are presented in Table I. The reaction system consisted of $2 \mu 1$ sense primer, $2 \mu 1$ anti-sense primer, $12.5 \mu 1 \mathrm{SYBR}^{\circledR}$ PCR Master mix, $2 \mu 1$ template cDNA $(25 \mathrm{ng} / \mu \mathrm{l})$ and double-distilled water to a final volume of $25 \mu 1$. PCR was performed for 40 cycles, and each cycle included denaturation at $95^{\circ} \mathrm{C}$ for $5 \mathrm{sec}$, annealing and extension at $60^{\circ} \mathrm{C}$ for $30 \mathrm{sec}$, which followed pre-denaturation at $95^{\circ} \mathrm{C}$ for $30 \mathrm{sec}$. The relative copy number was analyzed by using the threshold crossing point $(\mathrm{Cq})$, which was calculated by the system's software, combining with the $2^{-\Delta \Delta \mathrm{Cq}}$ calculations (23).

Statistical analysis. Statistical analysis was conducted with one-way analysis of variance followed by a Tukey's post-hoc test using SPSS v.22 (IBM Corp., Armonk, NY, USA). P<0.05 was considered to indicate a statistically significant difference.

\section{Results}

GLP-1 induces M2 polarization and inhibits $M 1$ polarization in RAW264.7 cells. In an attempt to elucidate the effects of GLP-1 on M1/M2 polarization in RAW264.7 cells with RT-qPCR analysis, macrophage-specific markers, including proinflammatory factors for M1 (iNOS, IL-6 and TNF- $\alpha$ ) and M2-specific genes IL-10, mannose receptor-1 (MRC-1), macrophage galectin-1 (MGL-1) and arginine-1 (ARG-1), were profiled. As presented in Fig. 1A, GLP-1 led to a decrease in the expression levels of M1-specific genes when compared with the control group $(\mathrm{P}<0.05)$, which were similarly reduced in cells pretreated with GLP-1 compared with those treated with LPS $(\mathrm{P}<0.05)$. GLP-1 augmented the expression levels of M2-specific genes (Fig. 1B). These results indicate that GLP-1 induces macrophage polarization toward M2 and inhibits M1 polarization.

GLP-1 induces STAT3 signal activation in RAW264.7 cells. In order to elucidate the molecular mechanisms underlying the effect of GLP-1 on macrophage polarization, the effect of STAT3 

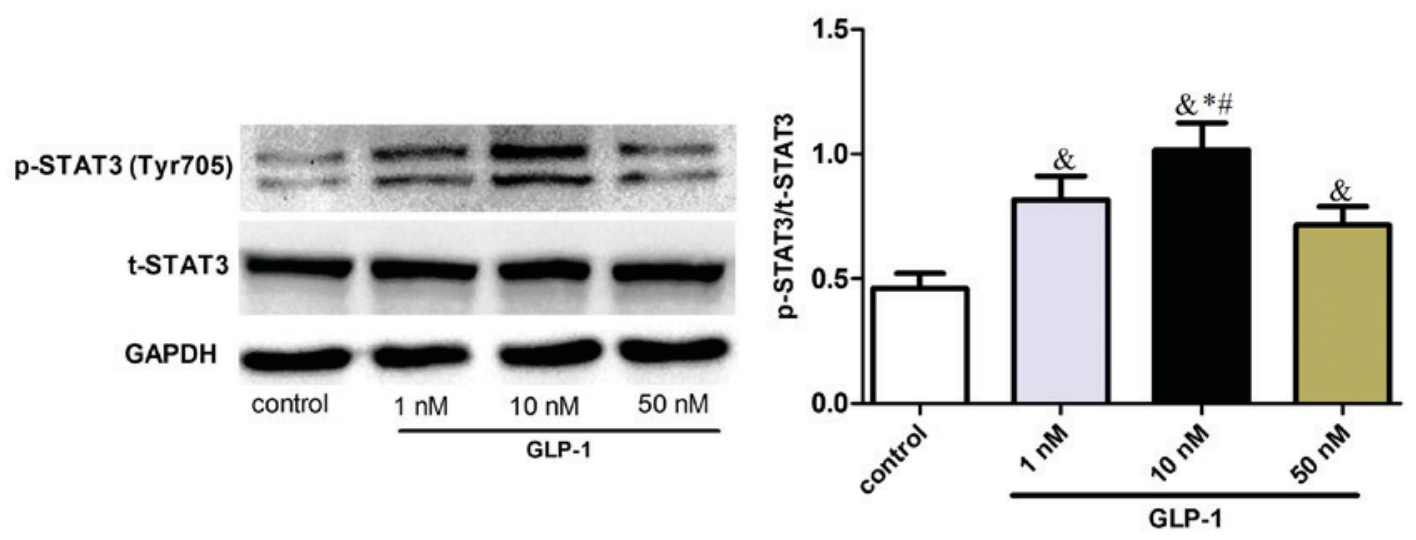

Figure 2. GLP-1 increases STAT3 activation in RAW264.7 cells. RAW 264.7 cells were incubated with various concentrations of GLP-1 (1-50 nmol/1) for 24 h. Cell extracts were prepared and analyzed by western blotting using anti-STAT3, anti-p-STAT3 and anti-GAPDH antibodies. The results are representative of three independent experiments. ${ }^{\&} \mathrm{P}<0.05$ vs. control group; ${ }^{*} \mathrm{P}<0.05$ vs. $1 \mathrm{nM}$ group; ${ }^{*} \mathrm{P}<0.05$ vs. $50 \mathrm{nM}$ group. GLP-1, glucagon-like peptide-1; STAT3, signal transduction and transcriptional activation factor 3 ; $\mathrm{p}$, phosphorylated; $\mathrm{t}$, total.
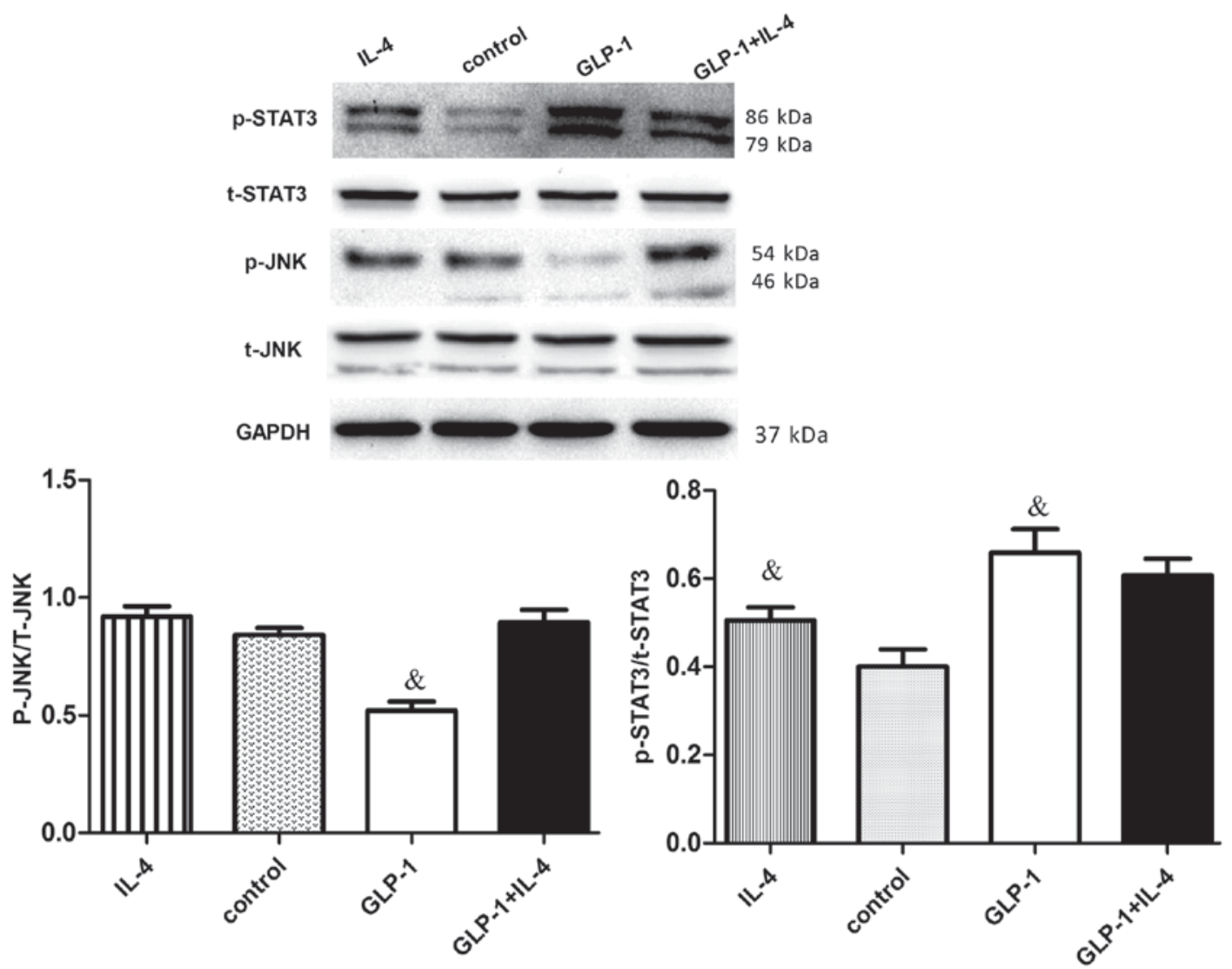

Figure 3. GLP-1 reduces the phosphorylation of JNK and increases the phosphorylation of STAT3 in RAW264.7 cells. RAW264.7 cells were incubated with or without $10 \mathrm{nM}$ GLP-1 for $24 \mathrm{~h}$, followed by IL-4 $(10 \mathrm{ng} / \mathrm{ml})$ for $24 \mathrm{~h}$. Cell extracts were prepared and analyzed by western blotting using anti-STAT3, anti-p-STAT3, anti-JNK, anti-p-JNK and anti-GAPDH antibodies. To determine the expression of phosphorylated JNK, JNK was used as the loading control, while the expression of phosphorylated STAT3 was determined using STAT3 as the loading control. The results are representative of three independent experiments. ${ }^{\circledR} \mathrm{P}<0.05$ vs. control group. GLP-1, glucagon-like peptide-1; STAT3, signal transduction and transcriptional activation factor 3; JNK, c-Jun N-terminal kinase; IL, interleukin; p, phosphorylated; t, total.

signaling on macrophage polarization toward M2 status was investigated in RAW264.7 cells. It was observed that GLP-1 upregulated the phosphorylation of STAT3 (Tyr705) (Fig. 2), suggesting that GLP-1 could induce STAT3 signaling activation and promote M2 activation of macrophages.

GLP-1 suppresses JNK phosphorylation activation and increases STAT3 signal activation in RAW264.7 cells. To elucidate the molecular mechanisms underlying STAT3 signaling activation by GLP-1, its inhibition effects on JNK were investigated. GLP-1 and IL-4 treatment increased STAT3 phosphorylation $(\mathrm{P}<0.05)$. However, JNK (Thr183/Tyr185) phosphorylation of GLP-1 was downregulated $(\mathrm{P}<0.05)$, while the total JNK protein levels were not significantly different (Fig. 3). Suppression of JNK phosphorylation accordingly upregulated the intracellular levels of phosphorylated STAT3 in RAW264.7 cells, suggesting that 


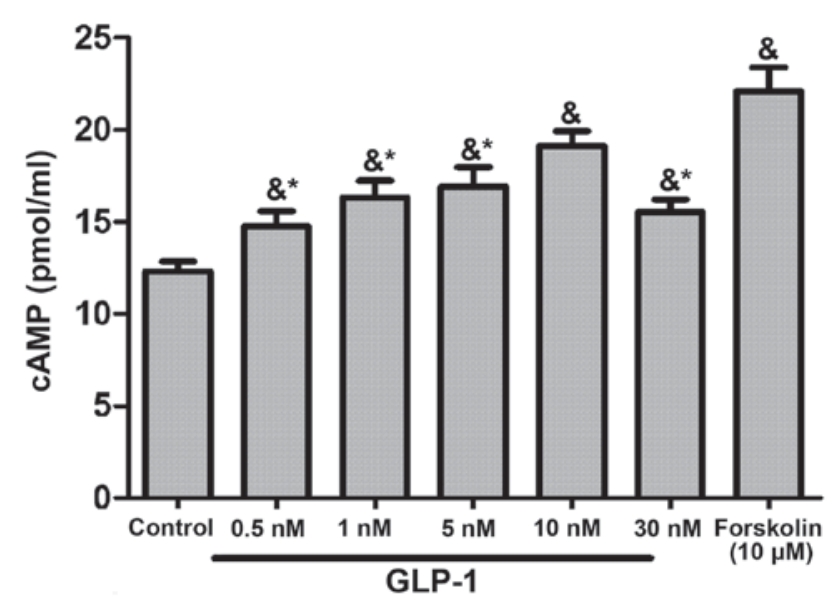

Figure 4. GLP-1 increases intracellular cAMP levels in RAW264.7 macrophages. RAW 264.7 cells were incubated with various concentrations of GLP-1 $(0.5-30 \mathrm{nmol} / \mathrm{l})$ for $8 \mathrm{~h}$. The Forskolin $(10 \mu \mathrm{M})$ group was set as the positive control. Cell extracts were prepared, followed by determination of the cAMP levels in RAW264.7 cells by enzyme immunoassay. The results are representative of three independent experiments. ${ }^{\&} \mathrm{P}<0.05$ vs. control group; ${ }^{*} \mathrm{P}<0.05$ vs. $10 \mathrm{nM}$ group. GLP-1, glucagon-like peptide-1; cAMP, cyclic adenosine monophosphate.
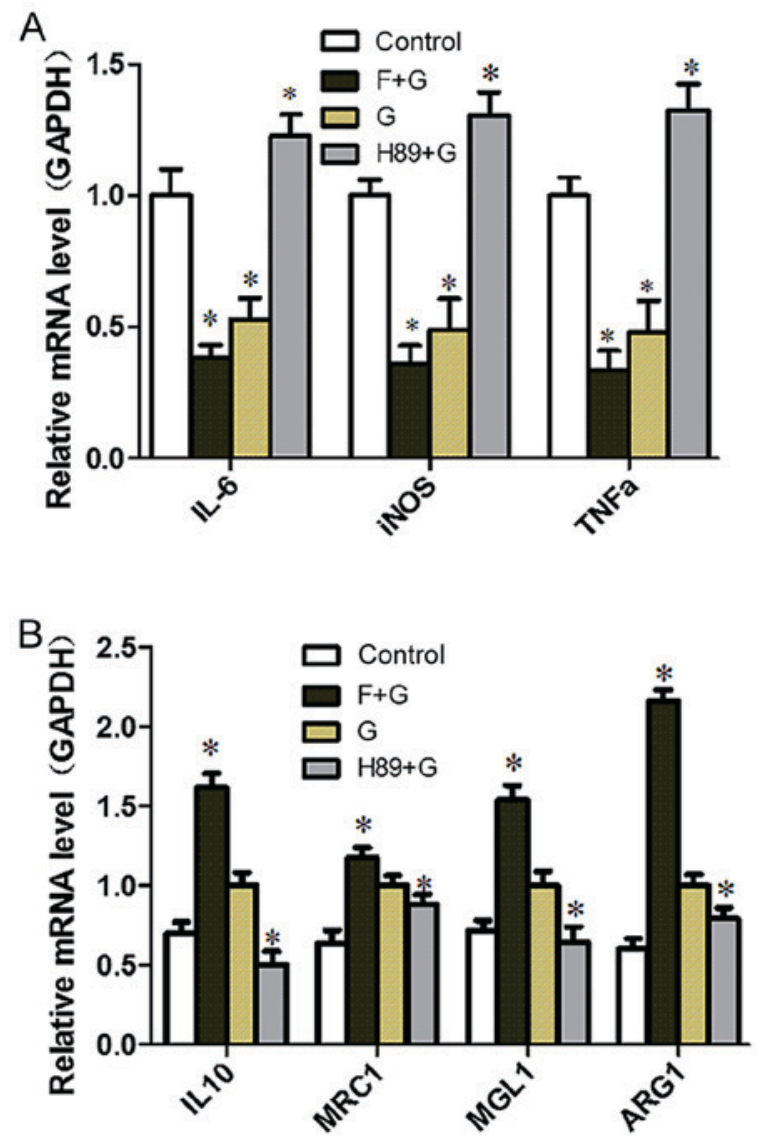

Figure 5. GLP-1/GLP-1 receptor signaling inhibits M1 activation and induces M2 activation via the cyclic adenosine monophosphate/PKA signaling pathway in RAW264.7 cells. (A) RAW264.7 cells were preincubated with the adenylyl cyclase activator Forskolin $(10 \mu \mathrm{M})$ or the PKA inhibitor H89 $(10 \mu \mathrm{M})$ for $30 \mathrm{~min}$, followed by treatment without or with GLP-1 $(10 \mathrm{nmol} / \mathrm{l})$ for $24 \mathrm{~h} .{ }^{*} \mathrm{P}<0.05 \mathrm{vs}$. control group. (B) RAW264.7 cells were preincubated with the adenylyl cyclase activator Forskolin $(10 \mu \mathrm{M})$ or the PKA inhibitor H89 $(10 \mu \mathrm{M})$ for $30 \mathrm{~min}$, followed by treatment with GLP-1 $(10 \mathrm{nmol} / \mathrm{l})$ for $24 \mathrm{~h}$. The mRNA expression levels of M1/M2-specific genes were determined by reverse transcription-quantitative polymerase chain reaction. The results are representative of three independent experiments. " P<0.05 vs. G group). GLP-1, glucagon-like peptide-1; PKA, protein kinase A; G, GLP-1 intervention; F, Forskolin intervention. the JNK-STAT3 signaling pathway could regulate macrophage polarization, resulting in counteraction between M1 and M2.

GLP-1/GLP-1R signaling inhibits $M 1$ activation and induces M2 activation by cAMP/PKA-mediated JNK downregulation in RAW264.7 cells. GLP-1 could increase cAMP levels in RAW264.7 cells in a concentration-dependent manner, with the most marked effect observed in the $10 \mathrm{nM}$ group ( $\mathrm{P}<0.05$; Fig. 4$)$. To identify the target molecule, the adenylate cyclase inhibitor Forskolin and the PKA inhibitor H89 were used. H89 pretreatment augmented the expression of M1-related inflammation genes $(\mathrm{P}<0.05)$ and reversed the inhibitory effect of GLP-1 on inflammatory factors $(\mathrm{P}<0.05)$ (Fig. 5A). Conversely, the expression of M2-specific genes was significantly increased with the addition of Forskolin, and was reduced with the addition of $\mathrm{H} 89(\mathrm{P}<0.05$; Fig. 5B); GLP-1 and Forskolin pretreatment reduced the expression of M1-related inflammation genes $(\mathrm{P}<0.05)$. These results suggest that GLP-1R signaling can promote M2 polarization and reduce $\mathrm{M} 1$ polarization through the cAMP/PKA signaling pathway, and JNK downregulation may serve a paramount role. Western blot analysis revealed that GLP-1 and Forskolin treatment suppressed the phosphorylation of JNK $(\mathrm{P}<0.05)$, while H89 treatment facilitated JNK phosphorylation $(\mathrm{P}<0.05)$ and blocked the inhibitory effect of GLP-1 on JNK phosphorylation $(\mathrm{P}<0.05)$ (Fig. 6). These results suggest that GLP-1/GLP-1R signaling inhibited JNK activation via the cAMP/PKA signaling pathway in RAW264.7 cells.

\section{Discussion}

The fact that macrophage activation acts on the development of inflammatory metabolic disorders such as insulin resistance and atherosclerosis is useful to investigate the mechanisms underlying insulin resistance. In the present study, GLP-1 was demonstrated to induce macrophage polarization toward M2 and inhibit M1 polarization. The present study elucidated that GLP-1R signaling contributes to the inhibition of JNK activation and leads to the activation of STAT3, which inhibits inflammation and M1 activation and promotes M2 activation. It was also demonstrated that GLP-1R signaling could promote M2 polarization and reduce M1 polarization through the cAMP/PKA-mediated JNK downregulation pathway. These findings suggest that modulation of signaling pathways may serve as pharmacological targets of macrophage activation and essential underlying mechanisms of GLP-1 on a broad spectrum of metabolic diseases.

Obesity and metabolic syndrome are accompanied by a phenotypic switch in the activation state of macrophages from an anti-inflammatory M2 polarization state to a proinflammatory M1 polarization state $(9,24,25)$. GLP-1 is known to improve insulin sensitivity, and may decrease macrophage infiltration and suppress the inflammatory response $(13,15)$. Consistent with the study of Shiraishi et al (16), it was confirmed that GLP-1 could increase the expression of M2-specific genes and induce the activation of STAT3, which is of paramount importance in macrophage differentiation toward the M2 phenotype. In addition, it was observed that GLP-1 could decrease M1-specific gene expression and inhibit M1 phenotype polarization, which were broadly congruent with the results of Arakawa et al and Chang et al $(13,22)$. Accordingly, prompting M2 polarization and suppressing M1 polarization may be effective treatments 
of insulin resistance and other inflammation-related diseases. However, little is known about how GLP-1/GLP-1R activates STAT3 signaling and the underlying mechanisms.

Activation of the stress-activated protein kinase/JNK serves an important role in macrophage polarization and insulin resistance. The JNK/mitogen-activated protein kinase pathways, located upstream of STAT3 signaling, also regulate STAT3 activity. In previous studies, JNK activation in Kupffer cells, peritoneal macrophages and adipose tissue macrophages led to M1 polarization, tissue inflammation and systemic insulin resistance (26-28). These data suggest that JNK expression in macrophages has a paramount importance on macrophage accumulation, M1 polarization and obesity-induced insulin resistance. The present study observed that GLP-1 inhibited JNK activation, which led to the reduction of M1 polarization and inflammatory factors, and accordingly ameliorated insulin sensitivity. Lim and Cao (29) proposed that dual regulation of STAT3 by JNK could upregulate serine phosphorylation and negatively regulate tyrosine phosphorylation. Overall, STAT3 serves a crucial role in M2 polarization, which mediates the anti-inflammation response (30-32). It was hypothesized that GLP-1/JNK/STAT3 signaling may induce macrophage polarization, which is in accordance with the inhibition of GLP-1 on JNK activation observed in the present study. The present results revealed that the effect of GLP-1 on STAT3 in RAW264.7 cells suppressed the effect of activated JNK on phosphorylated STAT3. Therefore, GLP-1 may suppress JNK phosphorylation and accordingly upregulate the intracellular levels of phosphorylated STAT3 in RAW264.7 cells, which promotes M2 polarization and is associated with anti-inflammatory effects. These results indicated that the JNK-STAT3 signaling pathway could regulate macrophage polarization, resulting in counteraction between M1 and M2.

Previous studies demonstrated that the main effects of GLP-1 are mediated through the activation of adenylate cyclase and the generation of cAMP, which subsequently activates PKA signal transduction $(22,33)$. The effects of increased intracellular cAMP levels in macrophages on inflammatory mediator generation were originally reported to be mediated by PKA, being cAMP a key event for macrophage activation toward the M2 phenotype (13,20-22). Regarding the cytoprotective action of GLP-1 on pancreatic $\beta$-cells, Ferdaoussi et al (34) proposed the potent inhibitory effect of GLP-1R activation via the cAMP/PKA signaling pathway on JNK activity as a major mechanism for preventing $\beta$-cells apoptosis. Thus, the present study revealed that GLP-1 increased intracellular cAMP levels in RAW264.7 cells within a certain range, in a concentration-dependent manner. The present study also demonstrated that GLP-1-cAMP/PKA was located upstream of JNK signaling and was involved in restraining JNK activity. This data suggested that GLP-1 promoted M2 polarization and inhibited M1 polarization through the cAMP/PKA pathway, whereby JNK activity served a vital role. As a result of JNK activity, M1 is inhibited, while STAT3 activity leads to M2 growth. M2 activation has been largely defined by its ability to antagonize and counteract M1 polarization and the inflammatory response, including insulin resistance $(4,35)$. The present findings suggest that there is crosstalk between M1 and M2, where the JNK-STAT3 signaling pathway is a major contributor and regulates the counterpoise between $\mathrm{M} 1$ and $\mathrm{M} 2$. The findings also suggest that the effects of GLP-1 in endocrine and metabolic
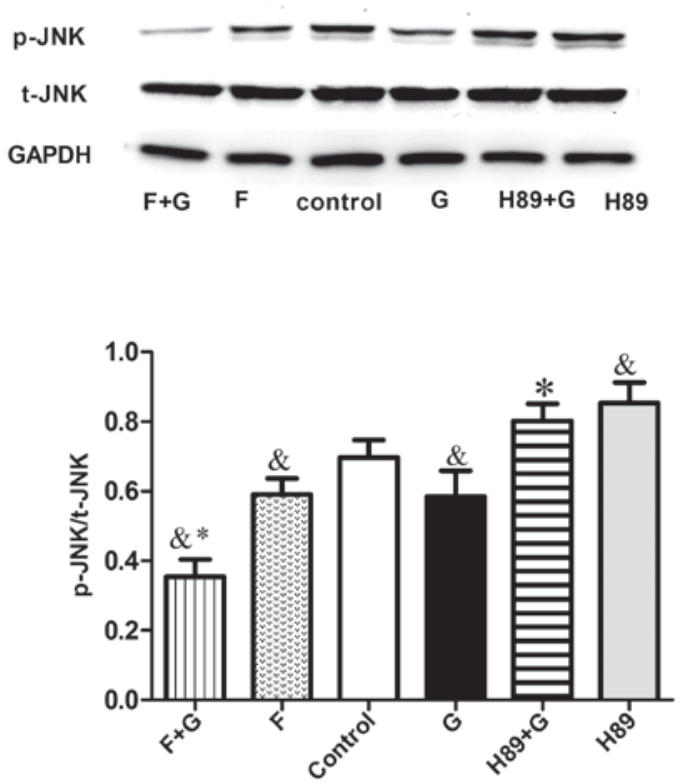

Figure 6. GLP-1 inhibits JNK activation via the cyclic adenosine monophosphate/PKA signaling pathway in RAW264.7 cells. RAW264.7 cells were preincubated with the adenylyl cyclase activator Forskolin $(10 \mu \mathrm{M})$ or the PKA inhibitor H89 $(10 \mu \mathrm{M})$ for $30 \mathrm{~min}$, followed by treatment without or with GLP-1 $(10 \mathrm{nmol} / \mathrm{l})$ for $24 \mathrm{~h}$. Cell extracts were prepared and analyzed by western blotting using anti-p-JNK, anti-JNK and anti-GAPDH antibodies. The results are representative of three independent experiments. ${ }^{\&} \mathrm{P}<0.05$ vs. control group; ${ }^{*} \mathrm{P}<0.05$ vs. G group. GLP-1, glucagon-like peptide-1; PKA, protein kinase A; JNK, c-Jun N-terminal kinase; G, GLP-1 intervention; F, Forskolin intervention; p, phosphorylated; t, total.

diseases are possibly mediated by the modulatory association of the signaling pathways, which provides insight into the molecular mechanisms behind the progression of metabolic diseases and may help to identify better drug targets. The therapeutic implications of this conclusion are notable as they suggest that pharmacological targeting of macrophage activation, rather than purely inflammation, may be efficacious in treating this global epidemic.

Due to the limitations of the present in vitro experiments, future investigations will focus on identifying factors that trigger the phenotypic switch of macrophage polarization using in vivo experiments and whether these changes in polarization state are generalizable to macrophages in tissues. Animal models are required to address these challenges, since these approaches allow specific deletion and cell type-specific strategies involved in macrophage polarization.

\section{Acknowledgements}

Not applicable.

\section{Funding}

The present study was supported by the Natural Science Foundation of Hubei Province, China (grant no. ZRY1073).

\section{Availability of data and materials}

The datasets used or analyzed during the current study are available from the corresponding author on reasonable request. 


\section{Authors' contributions}

HS conceived and designed the study. SW performed the experiments and wrote the paper. HS reviewed and edited the manuscript. All authors read and approved the manuscript.

\section{Ethics approval and consent to participate}

Not applicable.

\section{Patient consent for publication}

Not applicable.

\section{Competing interests}

The authors declare that they have no conflict of interest.

\section{References}

1. Gregor MF and Hotamisligil GS: Inflammatory mechanisms in obesity. Annu Rev Immunol 29: 415-445, 2011.

2. Aroor AR, McKarns S, Demarco VG, Jia G and Sowers JR: Maladaptive immune and inflammatory pathways lead to cardiovascular insulin resistance. Metabolism 62: 1543-1552, 2013.

3. Sica A and Mantovani A: Macrophage plasticity and polarization: In vivo veritas. J Clin Invest 122: 787-795, 2012.

4. Goerdt S and Orfanos CE: Other functions, other genes: Alternative activation of antigen-presenting cells. Immunity 10: 137-142, 1999.

5. Nathan C and Ding A: Nonresolving inflammation. Cell 140: $871-882,2010$.

6. Gordon S and Taylor PR: Monocyte and macrophage heterogeneity. Nat Rev Immunol 5: 953-964, 2005.

7. Mantovani A, Sica A, Sozzani S, Allavena P, Vecchi A and Locati M: The chemokine system in diverse forms of macrophage activation and polarization. Trends Immunol 25: 677-686, 2004.

8. Dall'Asta M, Derlindati E, Ardigò D, Zavaroni I, Brighenti F and Del Rio D: Macrophage polarization: The answer to the diet/inflammation conundrum? Nutr Metab Cardiovasc Dis 22: 387-392, 2012.

9. Odegaard JI and Chawla A: Alternative macrophage activation and metabolism. Annu Rev Pathol 6: 275-297, 2011.

10. Yabe D and Seino Y: Two incretin hormones GLP-1 and GIP: Comparison of their actions in insulin secretion and beta cell preservation. Prog Biophys Mol Biol 107: 248-256, 2011.

11. Friedrichsen BN, Neubauer N, Lee YC, Gram VK, Blume N, Petersen JS, Nielsen JH and Møldrup A: Stimulation of pancreatic beta-cell replication by incretins involves transcriptional induction of cyclin D1 via multiple signalling pathways. J Endocrinol 188 : 481-492, 2006.

12. Drucker DJ: The biology of incretin hormones. Cell Metab 3: 153-165, 2006.

13. Arakawa M, Mita T, Azuma K, Ebato C, Goto H, Nomiyama T, Fujitani Y, Hirose T, Kawamori R and Watada H: Inhibition of monocyte adhesion to endothelial cells and attenuation of atherosclerotic lesion by a glucagon-like peptide-1 receptor agonist, exendin- 4 . Diabetes 59: 1030-1037, 2010.

14. Li L, Yang G, Li Q, Tan X, Liu H, Tang Y and Boden G: Exenatide prevents fat-induced insulin resistance and raises adiponectin expression and plasma levels. Diabetes Obes Metab 10: 921-930, 2008.

15. Lee YS, Park MS, Choung JS, Kim SS, Oh HH, Choi CS, Ha SY, Kang Y, Kim Y and Jun HS: Glucagon-like peptide-1 inhibits adipose tissue macrophage infiltration and inflammation in an obese mouse model of diabetes. Diabetologia 55: 2456-2468, 2012

16. Shiraishi D, Fujiwara Y, Komohara Y, Mizuta H and Takeya M: Glucagon-like peptide-1 (GLP-1) induces M2 polarization of human macrophages via STAT3 activation. Biochem Biophys Res Commun 425: 304-308, 2012.
17. Takaishi K, Komohara Y, Tashiro H, Ohtake H, Nakagawa T, Katabuchi $\mathrm{H}$ and Takeya M: Involvement of M2-polarized macrophages in the ascites from advanced epithelial ovarian carcinoma in tumor progression via Stat3 activation. Cancer Sci 101: 2128-2136, 2010.

18. Yu H, Pardoll D and Jove R: STATs in cancer inflammation and immunity: A leading role for STAT3. Nat Rev Cancer 9: 798-809, 2009.

19. Williams L, Bradley L, Smith A and Foxwell B: Signal transducer and activator of transcription 3 is the dominant mediator of the anti-inflammatory effects of IL-10 in human macrophages. J Immunol 172: 567-576, 2003.

20. Serezani CH, Ballinger MN, Aronoff DM and Peters-Golden M: Cyclic AMP: Master regulator of innate immune cell function. Am J Respir Cell Mol Biol 39: 127-132, 2008.

21. Mustafa SB and Olson MS: Expression of nitric-oxide synthase in rat Kupffer cells is regulated by cAMP. J Biol Chem 273: 5073-5080, 1998.

22. Chang SY, Kim DB, Ryu GR, Ko SH, Jeong IK, Ahn YB, Jo YH and Kim MJ: Exendin-4 inhibits iNOS expression at the protein level in LPS-stimulated Raw264.7 macrophage by the activation of cAMP/PKA pathway. J Cell Biochem 114: 844-853, 2013.

23. Livak KJ and Schmittgen TD: Analysis of relative gene expression data using real-time quantitative PCR and the 2(-Delta Delta C(T)) method. Methods 25: 402-408, 2001

24. Weisberg SP, McCann D, Desai M, Rosenbaum M, Leibel RL and Ferrante AW: Obesity is associated with macrophage accumulation in adipose tissue. J Clin Invest 112: 1796-1808, 2003.

25. Lumeng CN, Bodzin JL and Saltiel AR: Obesity induces a phenotypic switch in adipose tissue macrophage polarization. J Clin Invest 117: 175-184, 2007.

26. Odegaard JI and Chawla A: Mechanisms of macrophage activation in obesity-induced insulin resistance. Nat Clin Pract Endocrinol Metab 4: 619-626, 2008.

27. Zhang X, Xu A, Chung SK, Cresser JH, Sweeney G, Wong RL, Lin A and Lam KS: Selective inactivation of c-Jun NH2-terminal kinase in adipose tissue protects against diet-induced obesity and improves insulin sensitivity in both liver and skeletal muscle in mice. Diabetes 60: 486-495, 2011.

28. Han MS, Jung DY, Morel C, Lakhani SA, Kim JK, Flavell RA and Davis RJ: JNK expression by macrophages promotes obesity-induced insulin resistance and inflammation. Science 339: 218-222, 2013.

29. Lim CP and Cao X: Serine phosphorylation and negative regulation of Stat3 by JNK. J Biol Chem 274: 31055-31061, 1999.

30. Li SN, Wang W, Fu SP, Wang JF, Liu HM, Xie SS, Liu BR, Li Y, Lv QK, Li ZQ, et al: IL-21 Modulates release of proinflammatory cytokines in LPS-stimulated macrophages through distinct signaling pathways. Mediators Inflamm 2013: 548073, 2013.

31. Matsukawa A, Kudo S, Maeda T, Numata K, Watanabe H, Takeda K, Akira S and Ito T: Stat3 in resident macrophages as a repressor protein of inflammatory response. J Immunol 175: 3354-3359, 2005.

32. Gaba A, Grivennikov SI, Do MV, Stumpo DJ, Blackshear PJ and Karin M: IL-10-mediated tristetraprolin induction is part of a feedback loop that controls Macrophage STAT3 activation and cytokine production. J Immunol 189: 2089-2093, 2012.

33. Makranz C, Cohen G, Reichert F, Kodama T and Rotshenker S: cAMP cascade (PKA, Epac, adenylyl cyclase, Gi, and phosphodiesterases) regulates myelin phagocytosis mediated by complement receptor-3 and scavenger receptor-AI/II in microglia and macrophages. Glia 53: 441-448, 2006.

34. Ferdaoussi M, Abdelli S, Yang JY, Cornu M, Niederhauser G, Favre D, Widmann C, Regazzi R, Thorens B, Waeber G and Abderrahmani A: Exendin-4 protects beta-cells from interleukin-1 beta-induced apoptosis by interfering with the c-Jun NH2-terminal kinase pathway. Diabetes 57: 1205-1215, 2008.

35. Gordon S: Alternative activation of macrophages. Nat Rev Immunol 3: 23-35, 2003.

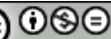

This work is licensed under a Creative Commons Attribution-NonCommercial-NoDerivatives 4.0 International (CC BY-NC-ND 4.0) License. 